\title{
Mehr Mitsprache bei Kontinenzprodukten
}

\author{
Lebensqualität und Zufriedenheit der Betroffenen lässt sich steigern
}

Etwa vier bis acht Prozent der Bevölkerung leiden unter Inkontinenz. Dennoch ist das Problem nach wie vor ein Tabuthema und schränkt die Lebensqualität der Betroffenen massiv ein. Ein besseres Verständnis und die Einbindung der Patienten in die Produktauswahl könnte die Zufriedenheit deutlich erhöhen. Allerdings: Es besteht Nachholbedarf, wie eine kürzlich veröffentlichte Nutzerbefragung des Global Forum on Incontinence (GFI) zeigt.

Bereits im vergangenen Jahr stellten acht führende europäische Patientenorganisationen sechs Empfehlungen auf, die die Versorgung von Menschen mit Inkontinenz verbessern könnten (1). So müsse die Kontinenzpflege als Menschenrecht anerkannt werden, um Betroffenen ein unabhängiges und würdevolles Leben $\mathrm{zu}$ ermöglichen, das Bewusstsein und das Verständnis für Inkontinenz unter Betroffenen und pflegenden Angehörigen müsse gesteigert und die Information über Inkontinenz und Kontinenzpflege verbessert werden. Auch die stärkere Einbeziehung von Menschen mit Inkontinenz in die Produktauswahl stellte eine der Empfehlungen dar. Die Ergebnisse der nun präsentierten europaweiten Studie bestätigen die Wichtigkeit dieser Komponente, zeigen aber auch, dass derzeit 25 Prozent der Befragten die von den Vertragspartnern der Krankenkassen zur Verfügung gestellten Inkontinenz-Produkte als ungeeignet empfinden, um aktiv am Alltag teilzunehmen. 43 Prozent hatten das Gefühl, die Produkte eigneten sich nicht, um sie während der Arbeit zu tragen. 41 Prozent fühlten sich von den Inkontinenz-Produkten im Schlaf gestört. Fast 40 Prozent bemängelten, dass sie keinen Einfluss auf die Produktauswahl hätten. 75 Prozent gaben an, dass sie zusätzliche Produkte aus eigener Tasche bezahlen müssen. Fazit: Damit Menschen mit Inkontinenz ein unabhängiges und würdevolles Leben führen können, müssen sie stärker in die Auswahl ihres Inkontinenz-Produkts einbezogen werden.

\section{Versorgung muss individuellen Bedürfnissen entsprechen}

„Aufgrund des demographischen Wandels wird es immer wichtiger, dass die Versorgung von inkontinenten Menschen ihren individuellen Bedürfnissen und Vorlieben entspricht", stellte Anne-Sophie Parent, Generalsekretärin der AGE Platform Europe fest. Bei der Pflege von Menschen mit Inkontinenz sei noch viel Spielraum für Verbesserungen vorhanden. „Betroffene könnten viel stärker in die Auswahl von Inkontinenz-Produkten einbezogen werden“, so Parent. Für eine größere Unabhängigkeit und Zufriedenheit im Alltag von inkontinenten Menschen hebt die Studie drei Schlüsselfaktoren hervor. Dazu gehören neben der stärkeren Einbeziehung in die Produktauswahl vor allem eine verbesserte Information und mehr Wissen über die verfügbaren InkontinenzProdukte sowie eine Erstattung der Krankenkassen, die sich an den individuellen Bedürfnissen des Betroffenen orientiert.

„Viele Betroffene wünschen sich Beratung zu aktiven Maßnahmen der Kontinenzförderung und natürlich auch zu Hilfsmitteln", erklärte die Pflegewissenschaftlerin Dr. Daniela Hayder-Beichel. In Deutschland werde diesem umfassenden Beratungs- und Unterstützungsbedarf jedoch zu selten nachgekommen. „Hier gilt es, neue Strategien im Gesundheitswesen zu entwickeln und erste gute Ansätze auszubauen.

\section{Ergebnisse der Nutzerbefragung}

- $41 \%$ fühlen sich durch die Produkte im Schlaf gestört

- $25 \%$ geben an, dass ihre Produkte ungeeignet sind, um aktiv am Alltag teilzunehmen

- $40 \%$ bemängelten, dass sie keinen Einfluss auf die Produktauswahl haben

- $75 \%$ müssen zusätzliche Produkte aus eigener Tasche bezahlen

- $43 \%$ haben das Gefühl, die Produkte eignen sich nicht, um sie während der Arbeit zu tragen

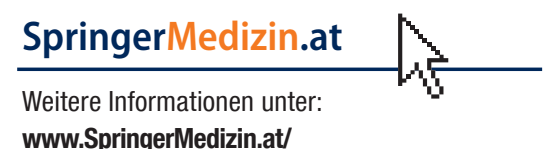

„Bei Inkontinenz stehen wir vor der Herausforderung, die Unabhängigkeit und das würdevolle Leben wiederherzustellen und betroffene Menschen bei der Teilnahme an der Gesellschaft zu unterstützen", so John Dunne, Eurocarers-Präsident. „Aus unseren Forschungsprojekten wissen wir, dass insbesondere intermittierende Inkontinenz in der häuslichen Pflege ein hoch relevantes Feld von Unterversorgung sein kann", erklärte Dr. Ralf Suhr, Vorstandsvorsitzender Stiftung Zentrum für Qualität in der Pflege (ZQP). Inkontinenz sei stark schambesetzt und sowohl bei den Betroffenen als auch bei den Helfern oftmals tabuisiert. „Auch dadurch wirkt Inkontinenz auf pflegebedürftige Menschen und ihre Angehörigen vielfach besonders belastend - sie gefährdet nicht zuletzt das Gelingen des häuslichen Versorgungssettings."

Die von der AGE Platform Europe - einem Netzwerk, das über 40 Millionen ältere Menschen in Europa vertritt - und dem Hygieneproduktehersteller SCA durchgeführte Untersuchung befragte Menschen mit Inkontinenz und pflegende Angehörige in Deutschland, Polen, England und Spanien.

Quelle: Pressemitteilung des Global Forum on Incontinence

\section{LITERATUR}

(1) http://bit.ly/1YW7osG 